\title{
Agriculture urbaine et développement durable : le cas des exploitants de la ceinture maraîchère de Brazzaville
}

\author{
André Moulemvo \\ Université Marien Ngouabi de Brazzaville
}

\section{Introduction}

D epuis le milieu des années 1980, avec la mise en œuvre des programmes d'ajustement structurel, le Congo connaît une montée de la pauvreté dans toutes ses dimensions et, depuis 2000, il est classé dans le groupe des pays à faible niveau de développement humain. La plupart des pauvres vivent dans les zones rurales, mais la pauvreté urbaine est elle aussi très prévalente et ne fait qu'augmenter. Chômage, faiblesse des revenus, faible accès aux soins de san-
La mise en œuvre d'un développement qui intègre des dimensions sociales, économiques et environnementales est un objectif auquel l'agriculture urbaine (AU) serait susceptible de contribuer.
La mise en œuvre d'un développement qui intègre des dimensions sociales, économiques et environnementales est un objectif auquel l'agriculture urbaine (AU) serait susceptible de contribuer. Mais, malgré les immenses possibilités de contribution qu'elle offre en termes de bien-être des générations actuelles et futures et d'écologie, son potentiel n'est que faiblement exploité. Elle est toujours sujette à de nombreuses contraintes, sa protection et sa promotion soulèvent plusieurs questions qui poussent des discours encore nombreux à la considérer comme irrémédiablement soumise au dété, au système éducatif et à l'eau potable sont autant de problèmes qui constituent le quotidien des citadins du Congo. Selon une étude menée à la fin des années 1990, $30 \%$ de la population urbaine du Congo vivent sous le seuil de pauvreté.

Brazzaville est pratiquement coupée de son hinterland, et en particulier de sa principale source d'approvisionnement, le département du Pool, du fait d'une dégradation poussée de la route nationale $\mathrm{n}^{\circ} 1$ et de la vétusté du Chemin de fer Congo océan (CFCO). Cette situation renchérit les vivres et aggrave la dépendance vis-à-vis des importations.

La croissance démographique rapide et l'activité économique de la ville engendrent des déchets dont le volume et la diversification posent des problèmes, tant de ramassage que d'élimination, à la municipalité dont les services techniques sont démunis. Cette situation a des conséquences désastreuses : dégradation des sites, enlaidissement de la ville, apparition de bourbiers malodorants, prolifération des mouches, rats, moustiques et autres vermines. clin par les forces du marché ${ }^{1}$. Aussi sommes nous amené à nous intéresser au cas particulier de Brazzaville et à nous poser deux questions, à savoir :

- Quels défis la ville de Brazzaville doit-elle relever pour la création en son sein et à sa périphérie d'un tissu de micros entreprises agricoles performantes et dynamiques ?

- Comment relever ces défis ?

L'objectif de notre article est de montrer l'impérieuse nécessité du développement à Brazzaville d'une AU performante et de dégager des esquisses de solutions aux nombreuses contraintes socio-économiques auxquelles elle fait face dans la perspective d'une pleine participation du secteur à l'objectif de développement durable. Au plan méthodologique, elle s'appuie sur une série d'entretiens avec les responsables de la direction générale de l'agriculture et de l'observatoire économique d'Agricongo ainsi qu'avec des exploitants maraîchers (en janvier-février 2005), mais aussi sur une recherche documentaire. 


\section{L'agriculture urbaine, facteur de développement durable}

\section{Documentation sur le rôle de l'agriculture urbaine dans le développement durable}

L'AU a fait l'objet de nombreuses études. Pour nombre d'entre elles, l'AU est un élément clé dans l'économie des ménages les plus démunis, constituant pour eux une source importante de nourriture et de revenus $^{2}$. Produire pour sa propre consommation en vue de réduire les dépenses d'alimentation auxquelles, selon Mougeot ${ }^{3}$, les ménages urbains pauvres consacrent entre $60 \%$ et $90 \%$ de leur revenu est la première motivation pour de nombreux urbains impliqués dans l'agriculture. En outre, en augmentant la diversité et la qualité des aliments consommées, l'AU peut améliorer significativement les régimes alimentaires urbains ${ }^{4}$.

L'AU offre une multitude de possibilités de création d'entreprises ${ }^{5}$. Elle pourrait en effet permettre le développement des entreprises de production, de transformation, des entreprises fournissant des matières premières et des services. L'AU pourrait à cet effet servir de point d'entrée des femmes et des jeunes dans l'entrepreneuriat et le marché du travail urbain ${ }^{6}$.

L'AU a des conséquences importantes en termes d'environnement. Nombre d'auteurs relèvent notamment l'impact positif qu'elle a sur l'environnement ${ }^{7}$. Les besoins élevés en matière organique de la plupart des productions maraîchères conduisent au développement d'une activité de recyclage des déchets urbains, contribuant à préserver le milieu de vie des citadins ${ }^{8}$.

\section{Les enjeux de l'agriculture urbaine à Brazzaville}

Reconnue comme essentielle pour l'accès d'une part croissante de la population urbaine des pays en développement aux denrées alimentaires, aux revenus, à l'emploi ainsi qu'à une politique environnementale, l'AU pourrait constituer, dans le cas particulier de la ville de Brazzaville :

- un levier de réduction de la pauvreté;

- une source d'approvisionnement de la ville en vivres frais;

- un moyen d'assainissement de la ville.

\section{Un levier de réduction de la pauvreté}

L'AU a une importance particulière pour le Congo qui connaît, depuis le milieu des années 1980, une paupérisation de sa population. Les résultats de trois études menées entre 1996 et 2003 indiquent que la proportion des personnes pauvres vivant avec moins de un dollar de revenu par jour se situerait à plus de $50 \%$, quelles que soient l'année étudiée et la méthode utilisée. En outre, le PNB par tête d'habitant, qui constitue une mesure fiable du revenu de vie des populations, n'a cessé de baisser au cours de la dernière décennie. Le Congo, autrefois considéré comme pays à revenu intermédiaire à coté du Gabon, du Cameroun et de la Cote d'Ivoire est, depuis 1995, classé parmi les pays à faible revenu. La faiblesse des revenus se traduit entre autres par une faible accessibilité aux systèmes éducatifs et sanitaires :

- Le Congo Brazzaville était, dans les années 19601970, placé parmi les pays les plus scolarisés de la planète, avec un taux officiel de près de $95 \%$. Actuellement, une proportion importante de la population est exclue du système éducatif. D'après le Rapport national sur le développement humain (2002), le taux de scolarisation des enfants de 6 à 14 ans n'était plus que de $73 \%$ en 1999. Le taux de scolarisation combiné (du primaire au supérieur) était quant à lui de $57 \%$ en 2001

- Selon le Programme des Nations Unies pour le développement (PNUD) (2003), une partie (20\%) de la population est privée d'accès aux soins de santé et aux médicaments essentiels et donc livrée aux guérisseurs traditionnels et aux marchands ambulants qui fournissent des soins de qualité douteuse. De nombreuses naissances ne sont pas suivies par le personnel de santé, ce qui cause un taux élevé de mortalité maternelle et infantile.

\section{L'AU a une importance particulière pour le Congo qui connaît, depuis le milieu des années 1980, une paupérisation de sa population.}

Comme conséquence, le Congo a enregistré au cours de la dernière décennie un important recul de ses deux indicateurs composites de développement humain calculés par le PNUD. L'indice de pauvreté humaine 
(IPH) est passé de 30,1\% en 1990 à 34,5\% en 2001 et à $31,9 \%$ en 2002 . L'indice de développement humain (IDH) est quant à lui passé de 0,507 en 2000 à 0,494 en 2002, plaçant du coup le Congo dans le groupe des pays à faible niveau de développement humain.

\section{En raison du vieillissement de sa population et des difficultés d'évacuation de ses produits, l'arrière-pays sera de plus en plus incapable de faire face aux besoins de la ville en produits alimentaires.}

Comme tout le reste du pays, la ville de Brazzaville connaît une paupérisation croissante de sa population. Même si, aujourd'hui, le niveau de vie y reste plus élevé qu'à la campagne, les tendances négatives tendent à avoir un impact beaucoup plus marqué. Une bonne partie des Brazzavillois éprouvent énormément de difficultés pour subvenir aux besoins élémentaires vitaux comme celui de se nourrir, de se soigner, voire de se loger. Une analyse sur Brazzaville dans le cadre de la rédaction du document intérimaire de stratégie de réduction de la pauvreté (2001) révèle une forte incidence de la pauvreté (plus de $30 \%$ ). Le taux de chômage est passé de 30,3\% en 1997 à 36,4\% en 2000 .

L'AU, d'où de nombreux ménages ( $25 \%$ selon Moustier) tirent la majeure partie de leurs revenus, pourrait, dans le cadre des interventions pour la réduction de la pauvreté, constituer une stratégie de survie essentielle pour les pauvres en leur procurant au moindre coût à la fois des emplois et des revenus. En effet, les petites exploitations de maraîchage peu exigeantes en capital de départ sont bien rentables. Une unité de maraîchage de 10000 FF (francs français) en création et en services d'appui crée dès la première année, pour un petit exploitant bien formé, plus de 24000 FF de revenu net ${ }^{9}$.

\section{Une source d'approvisionnement de la ville en vivres frais}

Le Congo est l'un des pays les plus urbanisés d'Afrique tropicale. La proportion de la population urbaine est passée de $32,84 \%$ en 1970 à $41,99 \%$ en 1980 puis à $66 \%$ en 2001 (la population de Brazzaville est passée de 585812 habitants en 1984 à 856410 habitants en 1996 et à près d'un million actuellement). L'extrapolation de ces tendances indique que plus de $80 \%$ des congolais vivront en ville en 2015 . L'accroissement rapide de la population urbaine n'est pas dû seulement à un accroissement naturel de la population urbaine, mais beaucoup plus à l'apport des populations venues des campagnes et des pays voisins, le Congo Kinshasa en particulier. Ces migrations vers les villes et leurs ceintures périphériques, qui s'expliquent pour une bonne part par la priorité accordée aux urgences des villes et par le manque d'intérêt pour le développement rural, aggravent le vide humain qui caractérise la campagne congolaise et entrainent le vieillissement de sa population. Couplées avec le manque de progrès technique (la quasi-totalité des paysans continuent à pratiquer une agriculture extensive et traditionnelle), ces tendances engendrent une stagnation, sinon une diminution des forces de production à la campagne.

D'autre part, on observe une aggravation des difficultés de communication entre Brazzaville et son hinterland auquel elle est reliée par cinq grandes voies (les routes nationales $\mathrm{n}^{\circ} 1$ et $\mathrm{n}^{\circ} 2$, le CFCO, la voie fluviale et la route de Mayama). Actuellement, seule la nationale $n^{\circ} 2$ joue pleinement son rôle, les quatre autres voies d'alimentation de Brazzaville étant pratiquement bouchées. La nationale $\mathrm{n}^{\circ} 1$ et la route de Mayama sont dans un état de dégradation très avancé. Le trajet Brazzaville Kinkala (chef-lieu du département du Pool) long de 77 kilomètres qui, autrefois, pouvait être parcouru en une heure au plus prend actuellement un ou deux jours. Le CFCO est vétuste et exsangue et se caractérise par un mauvais état des infrastructures, une insuffisance de matériel de traction et de remorques. Il n'assure plus que trois à quatre trains vers Brazzaville par semaine, ce qui se traduit par une régression continuelle de son trafic de marchandises (1212900 tonnes en 1985,236400 en 2000 et 717040 en 2002). La voie navigable est aussi en état de délabrement (mauvais état des voies, des infrastructures, des unités flottantes et des installations portuaires).

Ainsi, en raison du vieillissement de sa population et des difficultés d'évacuation de ses produits, l'arrièrepays sera de plus en plus incapable de faire face aux besoins de la ville en produits alimentaires. La ville de Brazzaville sera de plus en plus dépendante d'une agriculture de proximité pour assurer son ravitaille- 
ment, particulièrement en légumes frais. Aujourd'hui déjà, la part des jardins situés dans la ville et dans sa périphérie proche représente $80 \%$ de l'approvisionnement en légumes-feuilles, produits très périssables et très importants dans les habitudes de consommation des congolais.

\section{Un moyen d'assainissement de la ville}

La croissance démographique accélérée et l'activité économique et sociale engendrent une production importante et diversifiée des déchets. Une étude menée au cours des années 1990 a estimé à $450 \mathrm{~g} /$ personne la production journalière de déchets, dont $48,54 \%$ de matières putrescibles. Devant l'impuissance des services municipaux de procéder au ramassage adéquat des déchets, plusieurs initiatives, parmi lesquelles le projet pilote de collecte et de valorisation des ordures ménagères de Brazzaville (nommé AVOBRA), ont vu le jour. Ce projet, qui visait la collecte de $25 \%$ des ordures ménagères produites chaque jour dans Brazzaville, la production de compost par enfouissement des matières fermentescibles et la vente du compost ainsi produit aux maraîchers, n'a pu être poursuivi du fait de la guerre civile. Actuellement, un service informel composé de jeunes gens fait le ramassage de porte à porte à l'aide de véhicules à traction humaine. Mais le problème d'insalubrité reste entier, car les moyens rudimentaires dont disposent ces jeunes ne permettent d'évacuer les déchets que vers la décharge sauvage proche. Le développement de l'AU, avec l'accroissement des besoins en matières organiques qu'il sous-entend, peut faire du ramassage et du recyclage des ordures des activités attractives et donc contribuer à l'assainissement de la ville. Aujourd'hui déjà, elle consomme la quasi-totalité des drèches de la brasserie de bière locale. De plus, l'AU peut contribuer à la protection des lieux vacants comme les abords des cours d'eau (Maboukoutsiekélé, Mfilou et Tsiémé).

\section{Contraintes au développement durable de l'agriculture urbaine a Brazzaville}

\section{Les problèmes fonciers}

Depuis l'indépendance, malgré l'explosion démographique, donc une forte demande, les pouvoirs publics n'ont développé aucun programme de construction de logements sociaux. Faute d'avoir été construits en hauteur, les vieux quartiers se sont avérés saturés, alors que leur densité ne dépassait pas 200 habitants à l'hectare. Les citadins, obligés de trouver eux-mêmes la solution au problème de logement, n'ont eu d'autre alternative que la migration vers la périphérie, donc de se tourner vers les propriétaires fonciers. Cela a entraîné une urbanisation sauvage et une extension spatiale considérable de la ville. Les habitations ont progressivement pris la place des périmètres de production maraîchère qui alimentaient la ville en légumes.

Les tentatives des gouvernements successifs d'obédience marxiste-léniniste de changer le régime foncier coutumier en décrétant la terre propriété de l'État (la loi n ${ }^{\circ} 52 / 83$ du 21 avril 1983) n'ont pas permis d'éradiquer le phénomène de vente anarchique de parcelles. Mais l'État, qui avait alors le pouvoir de mettre en défens des zones de production maraîchère, a, au contraire, contribué au rétrécissement, voire à la disparition de plusieurs de ces zones. L'exemple le plus frappant est celui de la zone maraîchère de Talangaï de 31 hectare à l'origine, créée par les autorités dans les années 1960 pour installer 200 maraîchers congolais expulsés de Kinshasa pour des raisons politiques. Autrefois principal fournisseur en légumes de la ville, elle a perdu environ 6 ha entre 1975 et 1985 du fait de l'installation successive d'une école, d'une brasserie, d'un hangar d'entreprise et même d'une plantation de manioc pour un groupe politique ${ }^{10}$.

La Conférence nationale souveraine de 1991 a rétabli les propriétaires fonciers dans leurs droits. Il s'est instauré depuis lors une cohabitation tendue entre droits coutumiers et droits constitutionnels. Des terres autrefois expropriées sont depuis lors revendues par les propriétaires fonciers, ce qui aggrave la précarité de la propriété foncière agricole. Les exploitants maraîchers qui, pour la plupart, ont accédé à la terre au moyen d'une installation anarchique, d'une installation par l'État, d'un versement de faibles sommes d'argent aux propriétaires fonciers ou encore d'un prêt sont sans protection face aux appétits des Brazzavillois nantis à la recherche de parcelles d'habitation. On assiste à une quasi-disparition de nombreux sites de maraîchage. Ainsi, au cours des dernières années, d'après les données de la Direction générale de l'agriculture :

- le site de Talangaï est passé de 24,5 ha à 11 ha, provocant un conflit entre les maraîchers qui, de 
par leur activité, l'ont amélioré et les propriétaires fonciers. Ce conflit fait actuellement l'objet d'une procédure judiciaire;

- le site de Kombé autrefois d'une superficie de 35 ha en a perdu 14;

- le site de l'ORSTOM est aujourd'hui réduit à 2,5 hectares;

- les 400 maraîchers du site de Mpila (appelé cents fils) sont en instance d'expulsion, le site ayant été choisi pour abriter une usine.

Une des conséquences de la pression foncière dans les sites intra urbains est le déplacement continu de l'activité maraîchère vers la périphérie de la ville, vers des sites plus éloignés des domiciles et parfois difficiles d'accès, augmentant considérablement les frais de transport et donc le coût de revient des légumes et entraînant une réduction des revenus des maraîchers (si, pour se rendre au site de Talangaï, par exemple, il était nécessaire de débourser 300 FCFA [franc de la communauté financière africaine], le déplacement vers Djiri, nouveau site sur la route du nord, exige 1000 FCFA quotidiennement)

\section{Le faible accès aux crédits}

Dans les pays d'Afrique subsaharienne, les banques sont des entreprises transférées qui correspondent à un mode culturel étranger aux sociétés autochtones ${ }^{11}$. Traditionnellement, les agriculteurs n'ont donc que rarement recours aux crédits bancaires pour financer leurs activités. L'accès au crédit est tout aussi limité du coté de l'offre, surtout avec la libéralisation du secteur bancaire mise en œuvre dans le cadre des programmes d'ajustement structurel des années 1980 et 1990. La liquidation des banques nationales (y compris la Banque nationale de développement du Congo) et leur remplacement par des banques commerciales privées à participation étrangère (COFIPA Investment Bank, Crédit lyonnais et Congolaise de banque) ont plus encore affaibli la relation banques TPE (très petites entreprises) agricoles. Même les nombreuses institutions de micro finance créées à la faveur de la libéralisation du système bancaire, pourtant avec l'ambition de lutter contre la pauvreté en finançant des activités de survie, ne permettent pas de relever le défi. Elles ne disposent pas des moyens de leur ambition et sont plus promptes à financer les activités de commerce, de services, considérant les activités agricoles comme ayant des risques importants, une rentabilité aléatoire. Leurs conditions d'octroi des crédits (présentation d'un justificatif de revenu ou d'un compte d'exploitation actuel et/ou prévisionnel, mais aussi des pièces d'identité des cautions) sont autant de barrières infranchissables pour la grande majorité des exploitants maraîchers À titre d'exemple, les Mutuelles congolaises d'épargne et de crédit (MUCODEC), plus grande institution de micro finance du pays $(78,07 \%$ des dépôts et $83,63 \%$ des crédits en 2003) n'ont consacré aux activités agricoles en 2004 que $2,18 \%$ des crédits (contre 58,27 \% aux événements sociaux, $14,71 \%$ à 1 'habitat et $15,34 \%$ au commerce) ${ }^{12}$. Le manque d'accès à des programmes de crédit et d'investissement limite la capacité des exploitants maraîchers d'accéder aux facteurs de production (intrants et équipements agricoles) aux coûts onéreux, donc de moderniser leur activité et d'augmenter la production et leurs revenus. De nombreux exploitants maraîchers sont notamment obligés de recourir à une autoproduction de semences.

\section{Une des conséquences de la pression foncière dans les sites intra urbains est le déplacement continu de l'activité maraîchère vers la périphérie de la ville.}

\section{Contraintes de commercialisation}

Traditionnellement, les légumes sont fréquemment utilisés dans la cuisine congolaise, ce qui montre l'importance de la demande de légumes. Mais la faiblesse des revenus limite la propension des brazzavillois à la consommation de légumes. Dans les familles généralement pauvres et nombreuses, on consomme beaucoup plus les légumes dits de base (amarante, choux chinois et oseille locale) bon marché mais ne garantissant aux maraîchers que des faibles marges. Les légumes de type européen (concombre, aubergines, carottes, ...), qui sont pourtant des produits à forte valeur ajoutée, sont peu fréquents dans la consommation quotidienne. Ils ne constituent, selon Moustier, que $5 \%$ des quantités consommées par les ménages congolais. La demande de ces légumes a chuté avec la réduction considérable du nombre d'Européens du fait de la crise économique et surtout de la guerre civile de 1997. De plus, les légumes produits à Brazzaville sont soumis à une rude concurrence des produits (choux, carottes, laitues, ciboule) venant de Kinshasa, vendus 
à des prix plus bas. À titre d'exemple, le kilo de choux vendu à 500 FCFA par les maraîchers de Brazzaville est proposé à $350 \mathrm{~F}$ par les Kinois.

Du fait de l'absence de système structuré de commercialisation des produits agricoles, les maraîchers vendent directement leurs produits aux femmes revendeuses qui, généralement, imposent leurs lois pour acheter à des prix dérisoires. En outre, l'absence de transmission de signaux du marché vers les producteurs ne permet pas à ceux-ci d'adapter leur offre à la demande.

\section{Contraintes climatiques}

Les zones de production maraîchère sont extrêmement dispersées dans la ville mais se situent généralement à proximité des cours d'eau (par exemple, le site de Talangaï est situé au bord du marécage du fleuve Congo, les sites de Mbimi et Agricongo au bord du fleuve Djoué). Les fortes pluies que Brazzaville connaît sur une bonne partie de l'année (d'octobre à mai) causent, en raison de cette proximité des cours d'eau, des inondations et des ensablements qui se traduisent souvent par des pertes énormes. Ces pluies occasionnent aussi des échecs de semis, des maladies et de la pourriture de légumes. En revanche, à la fin de la saison sèche, de nombreuses exploitations éloignées des cours d'eau souffrent d'un manque d'eau et des fortes chaleurs.

\section{Contraintes institutionnelles}

Alors qu'elle constitue une activité d'apport très importante pour de nombreux habitants de la ville, l'AU souffre d'un déficit d'attention de la part des autorités municipales et n'est pas prise en compte dans l'élaboration des options du développement local de Brazzaville. À titre d'exemple, le document intérimaire de stratégie de réduction de la pauvreté (2004) ne retient pas l'AU comme axe de réduction de la pauvreté.

\section{Autres contraintes}

L'environnement organisationnel dégarni des exploitants maraîchers (absence d'organisation syndicale) ne leur confère aucun mode de pression pour défendre leurs intérêts face aux autorités, aux propriétaires fonciers et aux revendeurs.

Une contrainte non moins importante est le vol de légumes dans les jardins qui concerne particulièrement

les légumes de type tempéré. Pour y faire face, les exploitants engagent des sentinelles ou encore s'organisent pour veiller à tour de rôle, ce qui n'est pas sans effet sur le coût de revient des légumes.

\section{Le programme ceinture maraîchère de Brazzaville, un modèle pour le développement de l'agriculture urbaine}

L'essor de l'AU exige que celle-ci occupe une plus grande place au sein des politiques de développement urbain et social au plan national, régional et local. $\mathrm{Au}$ Congo et dans les autres pays en développement, l'expérience du programme «Ceinture maraîchère de Brazzaville » mis en œuvre par Agricongo peut constituer une source d'inspiration.

Agricongo, association de droit congolais, créée en 1986 par Agrisud, a lancé, avec le concours financier de l'Union européenne, la Coopération française et l'État congolais, un programme de développement périurbain nommé Programme «Ceinture Maraîchère de Brazzaville» $(\mathrm{PCM})$. Conçu comme un véritable incubateur des TPE agricoles, le PCM visait, pour la période de 1995 à mi-2001, l'installation de 300 exploitations maraîchères, 20 élevages associés et unités de traitement des ordures ménagères, avec voies d'accès et structures de commercialisation, ainsi que la mise en place d'un observatoire économique et l'animation de la Filière maraîchère de Brazzaville.

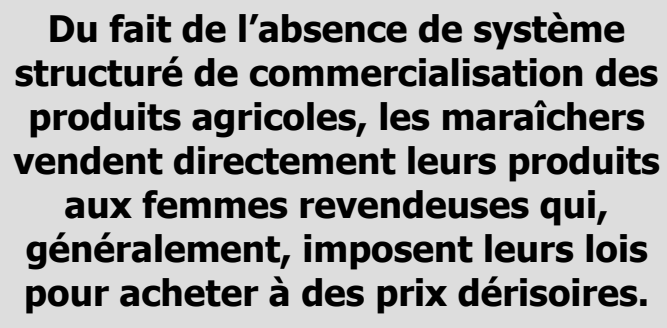

\section{Le projet ceinture maraîchère de Brazzaville : une approche innovante}

Le programme a mis au point un dispositif d'appui au développement agricole périurbain qui mise sur la sécurisation et la pérennisation de la profession, sur l'accompagnement des maraîchages, l'organisation des exploitants, l'information et la formation. Il est innovant à plusieurs titres : 
- Le PCM a apporté un changement majeur dans le paysage économique congolais en ce qu'il a prôné un appui à l'initiative privée dans un pays qui, jusqu'à la fin des années 1980, vivait une approche «tout État» issue d'une très longue époque marxiste basée sur une économie dirigée. Pendant cette période, l'initiative individuelle n'était « tolérée » que dans le cadre strict des organisations (groupements «pré-coopératifs») montées par l'État.

- Le PCM a mis au point et testé une procédure de création des micros entreprises qui se compose d'une étape de formation et de stage à l'issue de laquelle sont sectionnés les maraîchers à installer. À l'installation, Agricongo, agissant en véritable incubateur, met à leur disposition une subvention pour l'acquisition du matériel, des engrais, des semences, etc.

- La pérennisation et la sécurisation des exploitations passe par un règlement de la question foncière qui, comme nous l'avons signifié plus haut, constitue la principale contrainte au développement de l'AU. Dans un contexte où l'expropriation pour utilité publique se heurte à une farouche résistance des propriétaires fonciers (ils menaçaient d'expulser les maraîchers du site Rive droite du Djoué, long de 5,3 km et profond de $300 \mathrm{~m}$, suite à la volonté de l'État d'user de son droit «officiel» de propriété par une mise en défens sans aucune contrepartie [décret 89/206 de 1989]), le PCM a opté pour une nouvelle approche, la négociation et la signature des contrats de location entre les autorités et les propriétaires fonciers. Au PK 45, Agricongo est parti encore plus loin en achetant des parcelles sur lesquelles sont installés les maraîchers.

- Un accord de partenariat, en vue d'un appui à la gestion de l'épargne et d'un accès au crédit, a été conclu avec les Mutuelles congolaises d'épargne et de crédit (MUCODEC). Ce partenariat sera d'ailleurs, dans un proche avenir, régi par un protocole actuellement en élaboration.

- Les maraîchers sont organisés en groupements (il y en a huit au total), ce qui permet un accès collectif à des biens que chacun d'eux n'aurait pas pu acquérir et entretenir individuellement (motopompe, château d'eau...) et la mise en place des systèmes d'irrigation et leur gestion.

- Les groupements ont constitué des fonds de garantie auprès des MUCODEC en vue de permettre à leurs adhérents d'avoir accès aux fonds de roulement dont ils ont parfois besoin pour faire face à certaines situations exceptionnelles. Ces fonds de garantie ont été tout dernièrement renforcés par le ministère de l'Agriculture en mettant à la disposition de chacun des groupements une somme de 800000 FCFA.

- Des filières d'approvisionnement en matière organique ont été structurées, notamment par la signature de contrats de fourniture de matière organique (ordures ménagères triées, fumiers de ferme, drèches de brasserie). Ainsi, le PCM a signé avec l'association Bopeto un contrat portant sur l'équivalent de 8000 brouettes d'ordures ménagères triées par an, au prix unitaire de 500 FCFA.

\section{Impact socio-économique du programme PCM}

L'impact du PCM peut être apprécié à plusieurs niveaux :

- Recevant entre 150 et 250 stagiaires par an, le projet a permis la formation de plusieurs agriculteurs disséminés à travers le pays : 60 ont été installées sur le site de Kombé, 260 sur le site Rive droite du Djoué, 13 au PK 45. Le PCM a lancé une véritable dynamique de l'AU et permis ainsi l'émergence d'une nouvelle classe d'agriculteurs dans un pays où les jeunes scolarisés, pour la plupart, ne voulaient pas exercer dans l'agriculture, domaine pour lequel le pays dispose pourtant d'énormes potentialités (les surfaces cultivées sont évaluées à $4,9 \%$ des surfaces cultivables). Aujourd'hui, les exploitants maraîchers sont pour la plupart (à près de $70 \%$ ) des jeunes de 20 à 40 ans. On note aussi une forte implication des femmes. Elles constituent $48 \%$ des exploitants maraîchers bénéficiaires du PCM.

- Lancé en 1995, le PCM avait déjà en 1996 reçu 558 stagiaires et participé à la création de 170 micros entreprises et de 255 emplois directs dans le secteur du maraîchage. Jusqu'en 2000, on notait la création de 7000 emplois et de 1150 TPE dans 
lesquelles l'apport de main-d'œuvre familiale est fréquent et où l'on note un important développement du travail salarié (une exploitation $=1,8$ actif permanent + travailleurs temporaires).

- Les techniques acquises par les exploitants maraîchers du PCM permettent de faire du maraîchage tout au long de l'année, alors que le maraîchage traditionnel est soumis à l'influence saisonnière et ne se pratique qu'en saison sèche, entre juin et septembre. Pratiquant sur des parcelles de 600 à 1000 $\mathrm{m}^{2}$ une agriculture intensive avec des niveaux de maîtrise des systèmes élevés et une irrigation parfaitement maîtrisée, les exploitants maraîchers de la Rive droite du Djoué et de Kombé jouent un rôle de plus en plus important dans l'approvisionnement de Brazzaville en légumes. Leur contribution s'élève à près de $24 \%$ contre près de $50 \%$ pour les 5 000-6 000 exploitants des sites hors programme PCM, les autres sources d'approvisionnement étant la ville voisine de Kinshasa (République démocratique du Congo) (13\% à $20 \%)$ et l'arrièrepays ${ }^{13}$. En termes de revenus, les micros entreprises des maraîchers installés par le PCM procurent des revenus nets moyens mensuels variant entre 75000 et 150000 FCFA, selon les exploitations. C'est là un revenu comparable à un bon salaire de fonctionnaire de l'État. En revanche, les micros entrepreneurs hors programme ne gagneraient qu'entre 35000 et $45000 \mathrm{FCFA}$, selon les exploitations, en haute saison (saison sèche), leur activité connaissant un ralentissement en basse saison (saison de pluie) en raison des contraintes climatiques.

\section{Des filières d'approvisionnement en matière organique ont été structurées, notamment par la signature de contrats de fourniture de matière organique (ordures ménagères triées, fumiers de ferme, drèches de brasserie).}

- En plus des actions mises en œuvre sur les groupements directement touchés par le programme, le PCM a dynamisé l'ensemble de la filière. En effet, des candidats formés mais non intégrés dans les groupements ont créé des exploitations qui tentent de reproduire les modèles d'Agricongo et des producteurs « hors programme » ont été intéressés par des actions d'accompagnement (formations ciblées, conseils technico-économiques, accès aux intrants et aux petits équipements). Ainsi, le PCM a touché directement $20 \%$ des exploitations maraîchères permanentes de Brazzaville et a eu des effets d'entraînement, par des appuis ponctuels, sur les autres (au total 1200 à 1400 maraîchers permanents dans l'urbain et le périurbain).

- Le PCM a permis l'émergence de nombreuses activités connexes (commerce amont-aval, aménagement, ramassage et tri des ordures ménagères, artisanat). La plus en vue de ces activités est la commercialisation assurée par des commerçantes détaillantes qui s'approvisionnent directement auprès des producteurs (sur les périmètres de production et sur des marchés de « carreau »).

\section{Conclusion}

L'AU est une activité d'importance capitale pour la ville de Brazzaville en raison des difficultés de communication entre celle-ci et son hinterland, d'un fort déséquilibre démographique entre ville et campagne du Congo et d'une paupérisation croissante du milieu urbain. Si l'AU a été pratiquée jusque là sans une politique appropriée ni de cadres législatifs et institutionnels, en raison des défis auxquels elle fait face, il est nécessaire de créer un environnement favorable en vue de l'intégrer de manière effective dans l'économie urbaine, dans les programmes de développement urbain.

La démarche d'Agricongo, dans le cadre du Projet ceinture maraîchère de Brazzaville, illustre un tournant et témoigne au moins d'une certaine reconnaissance de l'AU. Cette démarche, qui s'articule autour d'une sécurisation du premier facteur de travail (la terre), d'une promotion des ressources humaines par la mise en œuvre des programmes de formation, d'un octroi de crédits par les institutions financières, etc., devrait être étendue pour couvrir des proportions de population encore plus importantes, ce qui sousentend une volonté politique des pouvoirs publics. L'octroi par ceux-ci au PCM d'un financement annuel prélevé sur les recettes pétrolières est à considérer comme un premier pas qui en appelle d'autres.

Agricongo a mis en place un dispositif de conseil de gestion et d'appui aux TPE créés dans le cadre du 
PCM. Ce dispositif, qui s'allège progressivement pour disparaître à la fin du programme, vise à l'acquisition d'une autonomie économique et sociale suffisante. Il est actuellement financé à parts égales par l'État et par les bailleurs de fonds (Coopération française et Union européenne), la contribution financière directe des bénéficiaires et de leurs organisations étant marginale. Le coût estimé de ce dispositif pour la période après PCM est de $5000 \mathrm{FCFA} / \mathrm{mois} /$ par exploitant, ce qui laisse présager une faible probabilité que les exploitants et leurs organisations assurent eux-mêmes la prise en charge financière du dispositif à un bon niveau. Aussi, la pérennité des structures d'appui mises en place est l'une des questions auxquelles il faudrait répondre. Un partenariat Etat-privéONG de développement pourrait à terme être envisagé pour assurer progressivement la relève des bailleurs de fonds étrangers dans le financement de l'incubation des TPE agricoles.

Pour le Congo resté longtemps sous un système « tout État », la création de micros entreprises sur des lopins individuels répond à la nécessité de promotion de l'initiative privée, mais cela n'exclut pas l'existence des « jardins collectifs » à l'image de ce qu'on observe dans les pays développés (au Québec, par exemple). Leur création pourrait contribuer à la promotion des ressources humaines. Comme le souligne Boulianne ${ }^{14}$, le travail en équipe sur un même espace et la participation à un plan de production commun faciliterait l'acquisition graduelle de compétences et des attitudes de constance et de ponctualité associées au monde de l'emploi.

\section{Notes biographiques}

André Moulemvo est Ph.D. en économie, assistant à la Faculté des sciences économiques de l'Université Marien Ngouabi de Brazzaville. Membre du Réseau Entrepreneuriat de l'Agence Universitaire de la Francophonie, il a participé aux IX ${ }^{\mathrm{e}}$ journées scientifiques du $1^{\text {er }}$ au 4 juin 2005 à Cluj Napoca, en Roumanie. Ce papier est une version révisée et actualisée de sa communication à ces Journées.

\section{Notes et références}

1 Moustier, P. et A. Salam Fall (2004). « Les dynamiques de l'agriculture urbaine : caractérisation et évaluation », Dé- veloppement durable de l'agriculture urbaine en Afrique francophone enjeux, concepts et méthodes, Canada, CIRAD/CRDI, CRDI.

2 Freeman, D.B. (1991). A City of Farmers : Informal Urban Agriculture the Open Spaces of Nairobi, Kenya, Montreal and Kingston, London, Buffalo McGill-Queen's University Press; Rakodi, C. (1988). «Urban Agriculture: Research Questions and Zambia Evidence ", Journal of Modern African Studies, vol. 26, n 3, p.495-515.

3 Mougeot, L. (1993), «Urban Food Reliance : Significance and Prospects », IDRC reports, vol. $21, \mathrm{n}^{\circ} 3$.

4 Ladame, P. (2005). La FAO pour des villes plus vertes, Rome, FAO.

5 FAO (1999). «Urban and Peri-urban Agriculture ». Food and Agriculture Organization of the United Nations, Rome, Italy. Committee on Agriculture Committee on Agriculture (15 $5^{\text {th }}$ Session). http://www.fao.org/unfao/bodies/ COAG/COAG15/X0076e.htm.

6 Freeman (1991), op. cit.

7 Moustier, P. et O. David (1997). Études de cas de la dynamique du maraîchage périurbain en Afrique subsaharienne, Rome, Italie, FAO, document N-DT/02/96.

8 Moustier et Salam Fall (2004), op. cit.

9 Baratier, J. (2003). « Entreprendre contre la pauvreté, l'expérience d'Agrisud International ", Enjeux du développement durable. Élément de débat, Paris, Karthala.

10 Moustier (1997), op. cit.

11 Baumann, E et A. Dia (1991). « Parle-moi d'argent ou Le théâtre Koteba au service de la Bank of Africa-Mali », $\mathrm{Ca}$ hier des sciences humaines, vol 27, n 3-4, p. 403-409.

12 Rakodi (1988), op. cit.

13 Moustier (1997), op. cit.

14 Boulianne, M. (2001). «Agriculture urbaine, au sein des jardins collectifs québécois : empowerment des femmes ou "domestication de l'espace public"»? Anthropologie et société économie politique féministe, vol. $25, \mathrm{n}^{\circ} 1$. 


\section{Publicité}

Centre de recherche sur le développement territorial

\section{CRDT}

